\title{
Using Aromatic Donor Acceptor Interactions to Affect Macromolecular Assembly.
}

\author{
Joseph J. Reczek and Brent L. Iverson*. \\ Department of Chemistry and Biochemistry, The University of Texas at Austin, Austin, \\ Texas \\ jreez@mail.utexas.edu, biverson@ mail.utexas.edu*
}

\section{Supporting Information.}

General methods. All starting materials were obtained from Aldrich and used without further purification. NMR spectra were taken on a Varian Unity +300 spectrometer in solutions of DMSO or 0.2M NaOD. Tapping-mode atomic-force microscopy (AFM) measurements were made using a Digital Instruments Dimension 3100 microscope in combination with a Nanoscope IV Controller (Veeco Metrology, Santa Barbara, CA). Scanning electron micrograph (SEM) data were obtained from a LEO 1530 scanning electron microscope operating at an accelerating voltage of $3 \mathrm{KeV}$. TGA experiments were performed on a TA Instruments TGA Q500. UV-Vis spectra were taken with a perkin-elmer lambda 35 spectrophotometer. $\sim 50 \mathrm{kD} \mathrm{M}_{\mathrm{w}}$ with a PDI of 2.2 polyethylenealt-maleic anhydride was supplied by the Zeeland chemical company. DSC experiments were performed on a TA Instruments DSC Q100, no transitions were observed in either of the polymers before degradation began at $250^{\circ} \mathrm{C}$. 


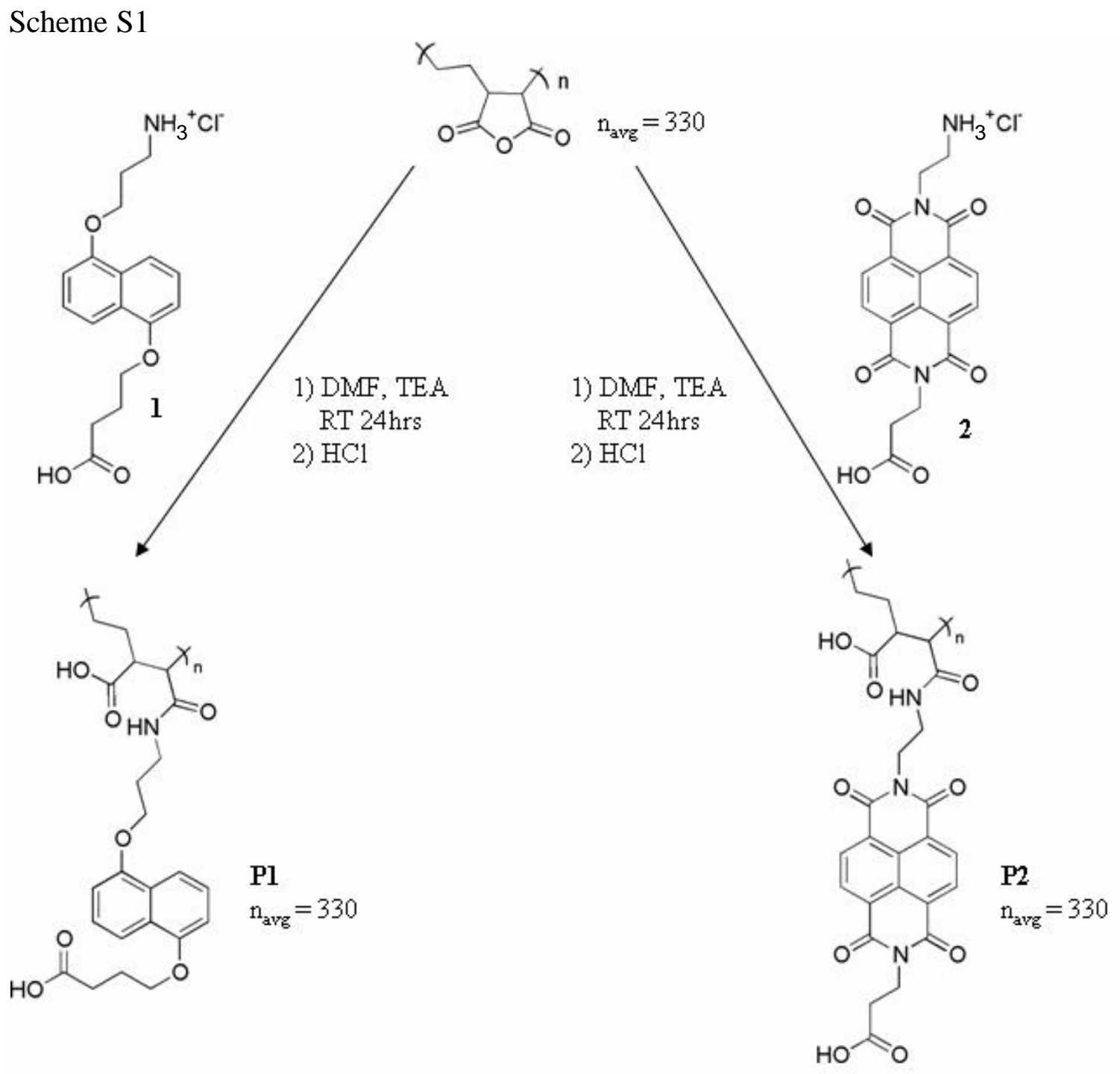

\section{Synthesis.}

PolyDan P1. Polyethylene-alt-maleic anhydride (0.6g, 4.76mmol anhydride) was dissolved in dry DMF (20ml) and TEA (1.44g, 14mmol) and placed under argon. 1 $(1.53 \mathrm{~g}, 4.69 \mathrm{mmol})$ in dry DMF $(20 \mathrm{ml})$ was added to the solution via addition funnel over 
$1 \mathrm{hr}$, and the reaction was allowed to stir at RT over night. Upon no evidence of reagent 1 by TLC in the reaction, the solution was precipitated in $1 \mathrm{M} \mathrm{HCl}$, filtered, and dried under vacuum at $30^{\circ} \mathrm{C}$ to yield $\mathbf{P 1}\left(1.2 \mathrm{~g}, 60 \%\right.$ recovered) as a light brown solid. ${ }^{1} \mathrm{H}$ NMR (0.5M NaOD + 0.01M SDS) $d 7.37$ (br, 2H), 6.85 (br, 2H), $6.36(\mathrm{br}, 2 \mathrm{H}), 4.04(\mathrm{br}, 2 \mathrm{H})$, 3.27 (br, 2H) $2.16(\mathrm{br}, 4 \mathrm{H}), 1.82(\mathrm{br}, 4 \mathrm{H}), 1.25(\mathrm{br}, 4 \mathrm{H})$

PolyNdi P2. Polyethylene-alt-maleic anhydride ( $0.6 \mathrm{~g}, 4.76 \mathrm{mmol}$ anhydride) was dissolved in dry DMF (20ml) and TEA (1.42g, 14mmol) and placed under argon. 2 $(1.88 \mathrm{~g}, 4.67 \mathrm{mmol})$ in dry DMF $(20 \mathrm{ml})$ was added to the solution via addition funnel over $1 \mathrm{hr}$, and the reaction was allowed to stir at RT over night. Upon no evidence of reagent 2 by TLC in the reaction, the solution was precipitated in $1 \mathrm{M} \mathrm{HCl}$, filtered, and dried under vacuum at $30^{\circ} \mathrm{C}$ to yield $\mathbf{P 2}$ (57\% recovered). ${ }^{1} \mathrm{H}$ NMR (DMSO) $d 12.39(\mathrm{br}, 2 \mathrm{H}$ ), $8.68(\mathrm{~s}, 4 \mathrm{H}), 4.29(\mathrm{t}, J=7.3 \mathrm{~Hz}, 2 \mathrm{H}), 3.33(\mathrm{br}, 2 \mathrm{H}), 2.61(\mathrm{t}, J=7.8 \mathrm{~Hz}, 2 \mathrm{H}), 2.52(\mathrm{t}, J=$ $9.3 \mathrm{~Hz}, 2 \mathrm{H}), 2.44$ (br, 2H), 1.42 (br, 4H) 
UV-Vis.

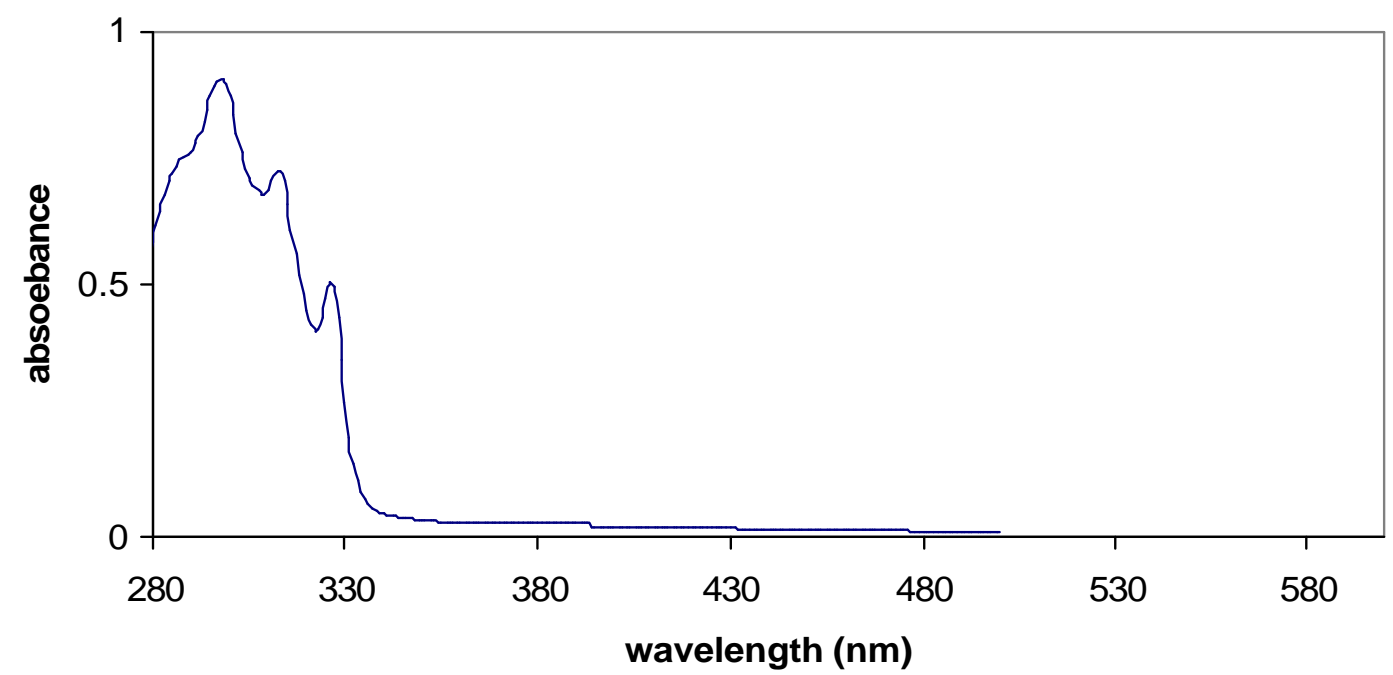

Figure S1. 0.6mM P1 + 0.01mM SDS detergent in 0.2 M NaOH. Sharp features of the Dan absorbance indicate little or no pi-pi interaction between electron rich Dan units.

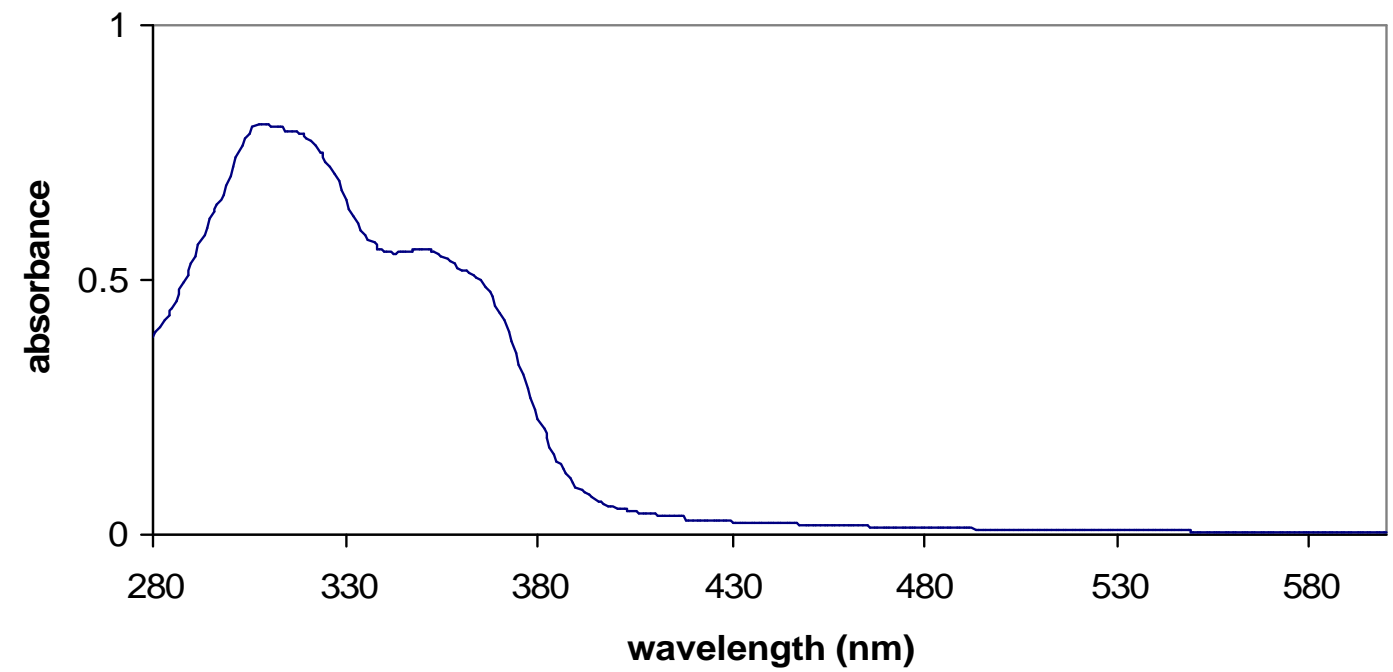

Figure S2. $0.6 \mathrm{mM}$ P2 in $0.2 \mathrm{M} \mathrm{NaOH}$. Lack of distinguishable features in the Ndi absorbance region of $340-400 \mathrm{~nm}$ indicates pi-pi self-association between Ndi units. 


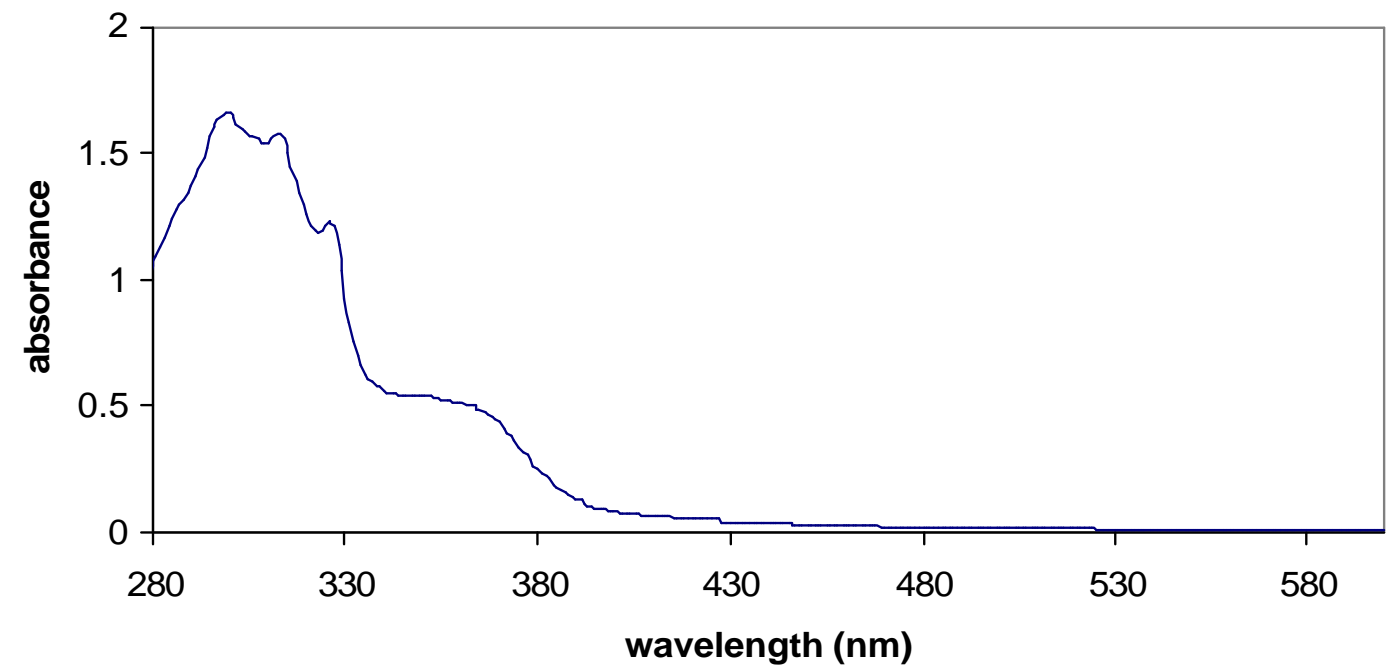

Figure S3. $0.6 \mathrm{mM} \mathrm{P1}+0.6 \mathrm{mM} \mathrm{P} 2$ in $0.2 \mathrm{M} \mathrm{NaOH}$. A small amount of hypochromism of the Ndi unit is observed with a slight red shift.

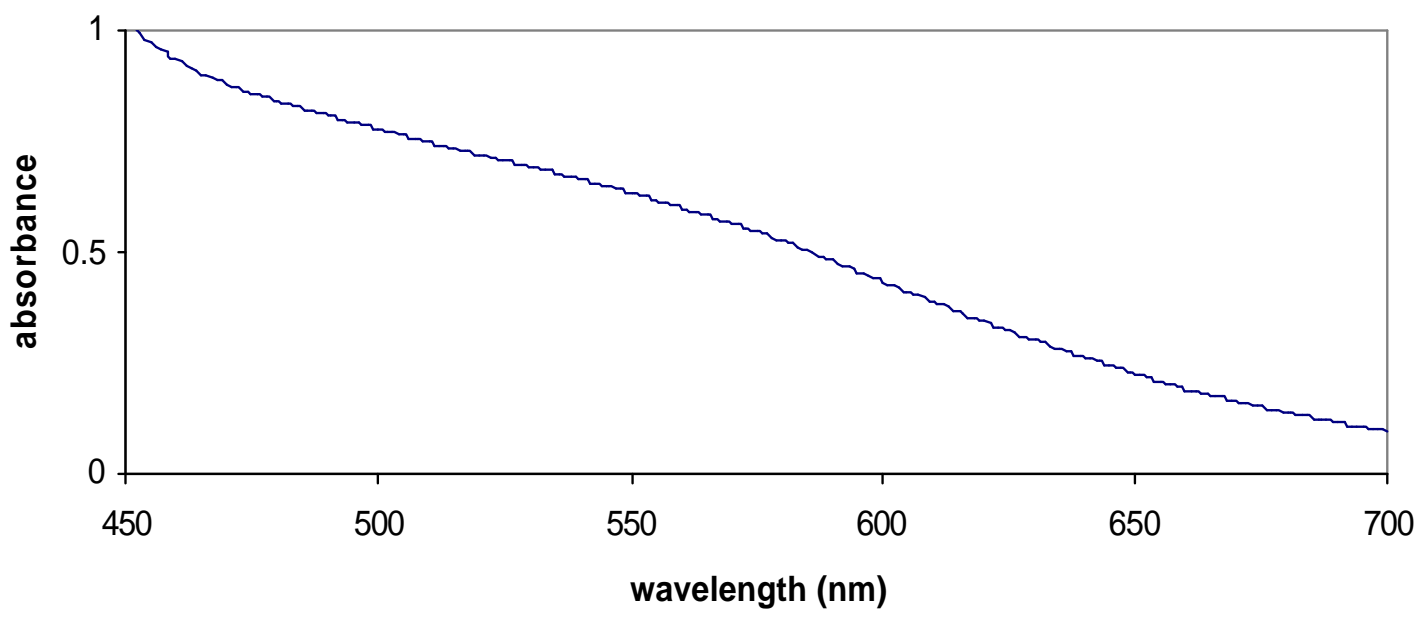

Figure S4. $6.0 \mathrm{mM} \mathrm{P} 1+6.0 \mathrm{mM} \mathrm{P} 2$ in $0.2 \mathrm{M} \mathrm{NaOH}$. A broad charge-transfer band is observed from 530-620nm, indicative of a Dan-Ndi face-to-face interaction. 


\section{TGA}
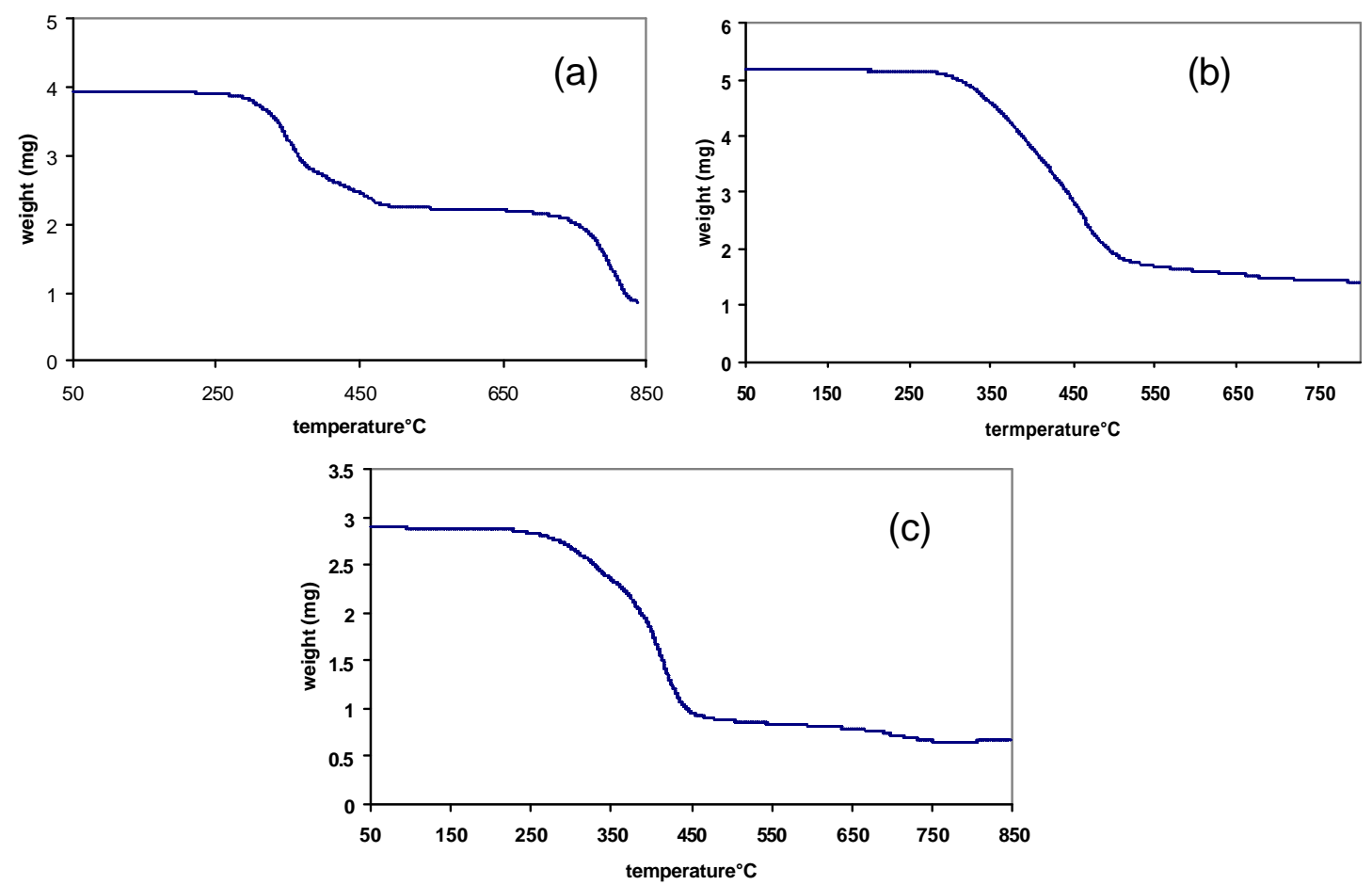

Figure S5. TGA analysis after precipitation and drying via vacuum oven at $70^{\circ} \mathrm{C}$ overnight. It likely that the amid rings on the polymer backbone close and water is driven off in the drying process. (a) P1+SDS, the observed shoulder is probobly due to trapped salts (b) P2 and (c) $\mathrm{P} 1+\mathrm{P} 2$ 
Dulue segrences sape

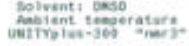

Relex deley 1.069 sot

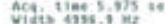

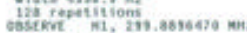

Hitanectsing

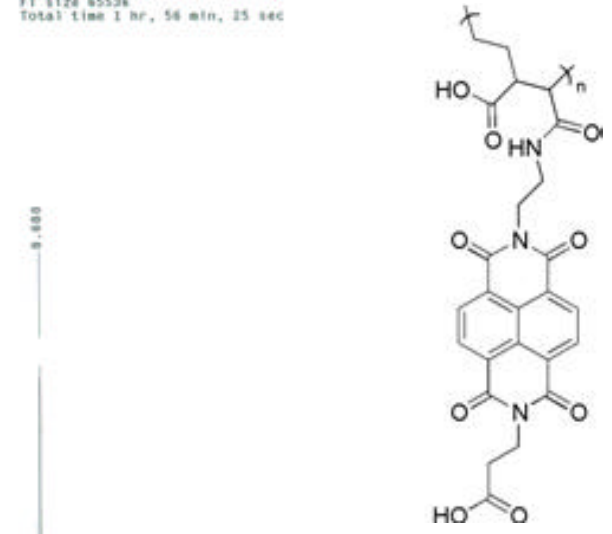

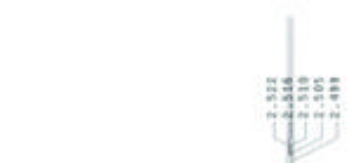

$\mathrm{H}_{2} \mathrm{O}$

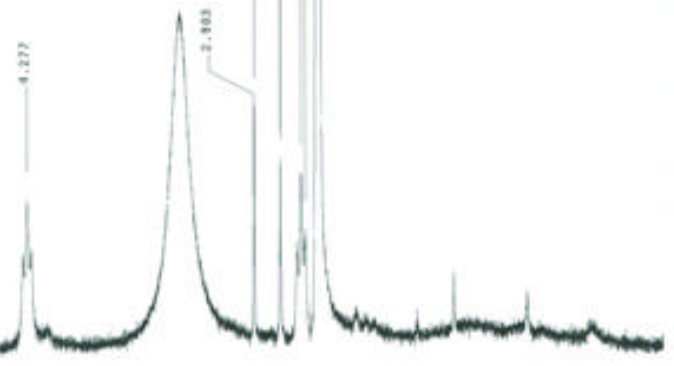




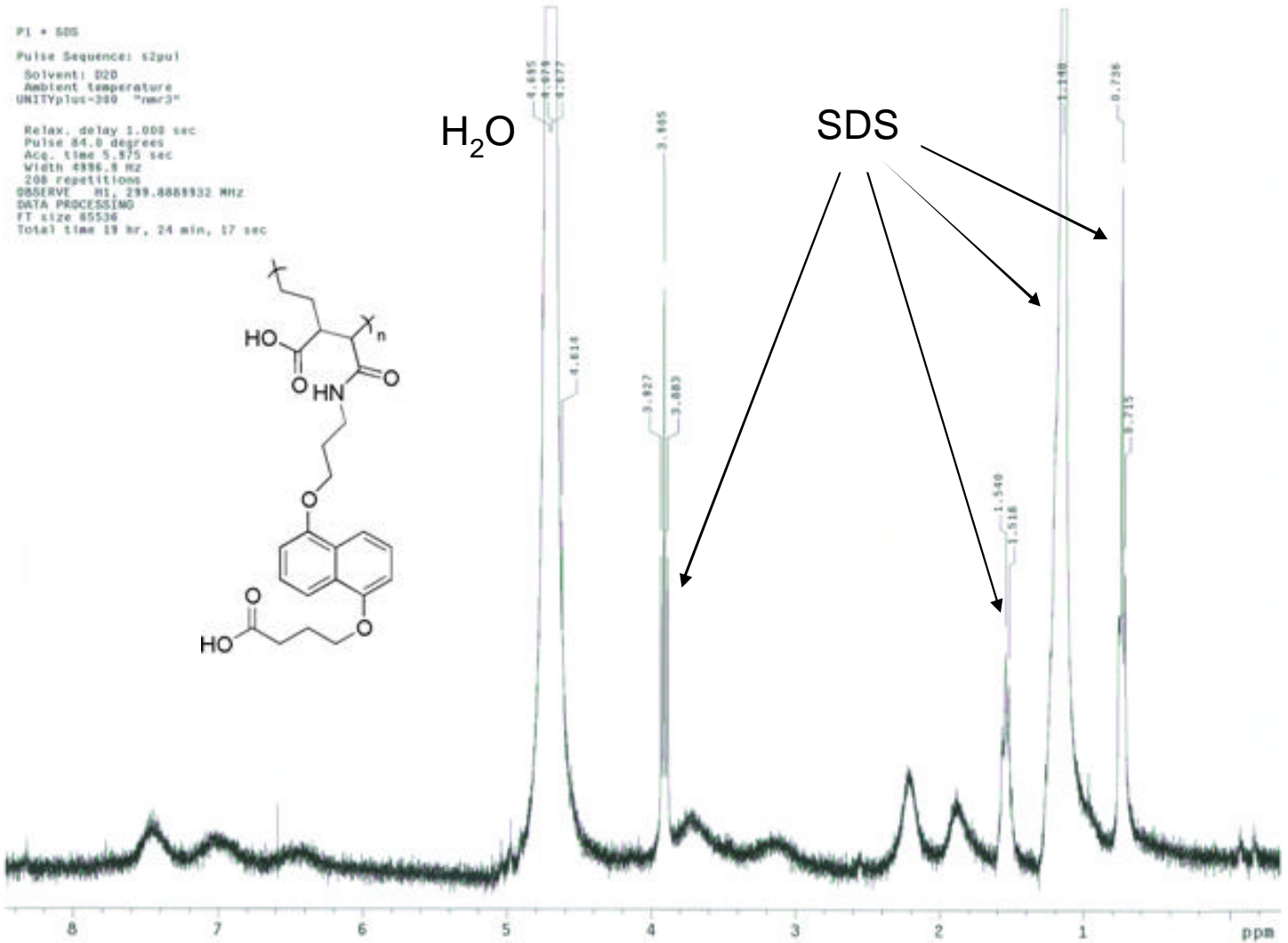

